\title{
Tangence
}

\section{Double public et complicité enfants-adultes dans le théâtre et le roman de Jasmine Dubé}

\section{Claire Le Brun}

Numéro 67, automne 2001

L'écriture pour la jeunesse : de la production à la réception

URI : https://id.erudit.org/iderudit/009619ar

DOI : https://doi.org/10.7202/009619ar

Aller au sommaire du numéro

Éditeur(s)

Presses de l'Université du Québec

ISSN

0226-9554 (imprimé)

1710-0305 (numérique)

Découvrir la revue

Citer cet article

Le Brun, C. (2001). Double public et complicité enfants-adultes dans le théâtre et le roman de Jasmine Dubé. Tangence, (67), 112-126.

https://doi.org/10.7202/009619ar
Résumé de l'article

Résumé

Dramaturge, metteure en scène et interprète, Jasmine Dubé a joué, dès le début des années 1980, un rôle de premier plan dans l'évolution du théâtre québécois pour la jeunesse. La prise en compte d'un double public, les enfants et «leurs» adultes, est au coeur de sa démarche théâtrale. Elle a également publié une série de romans destinés aux enfants de sept à neuf ans, les «Nazaire» (1994-1999). L'analyse des dialogues de cette série montre que Dubé transpose à l'écriture romanesque sa conception de l'écri- ture théâtrale, offrant, pour une lecture conjointe de l'enfant et de l'adulte parent, une mise en mots des situations délicates de la vie familiale. 


\section{Double public et complicité enfants-adultes dans le théâtre et le roman de Jasmine Dubé}

Claire Le Brun, Université Concordia

La présente analyse s'inscrit dans le prolongement d'une étude sur le théâtre pour jeunes publics de Jasmine Dubé, parue en 1997 dans l'ouvrage collectif Nouveaux regards sur le théâtre québécois ${ }^{1}$. Ce travail suivait la démarche thêâtrale de l'auteure de 1982 à 1996, en montrant comment elle créait, pièce après pièce, les conditions d'un dialogue, afin de "raccorder l'adulte et l'enfant ${ }^{2}$. Alors qu'il met en scène les réelles difficultés des deux groupes d'âge à entrer en phase, le théâtre de Dubé donne en effet la parole à des adultes ouverts et transparents, capables de respecter les besoins de leurs enfants tout en s'ouvrant à eux de leurs besoins propres et de leurs manques.

Dans le cadre d'une recherche sur les publics de l'écrivain ou de l'écrivaine pour la jeunesse, le cas de Jasmine Dubé est particulièrement digne d'attention. Tout en ceuvrant à diverses productions culturelles pour enfants, elle fait partie de ces auteurs qui se tournent résolument vers leur public. Mais il y a plus: la prise en compte d'un double public est véritablement au cœur de son projet littéraire. D'abord auteure dramatique, Dubé transpose sa conception de l'écriture théâtrale pour jeunes publics au roman pour lecteurs débutants. C'est ce que je me propose d'établir ici, en analysant la série des Nazaire, parue de 1994 à 1999 dans la collection "Premier Roman" à La courte échelle. Après un retour sur quelques pièces particulièrement éclairantes pour mon propos, je centrerai mon analyse sur les dialogues romanesques, lieux d'une communication significative entre l'enfant et l'adulte parent.

1. Claire Le Brun, "Raccorder l'adulte et l'enfant. Les voix des enfants et de leurs adultes dans le théâtre pour jeunes publics de Jasmine Dubé x, dans Betty Bednarski et Irène Oore, Nouveaux regards sur le théâtre québécois, Montréal/Halifax, XYX éditeur/Dalhousie French Studies, coll. "Documents ", 1997, p. 169-183.

2. Préface de la pièce Pierrette Pan, qui sera commentée plus bas. 


\section{À travers une œuvre protéiforme, une conception de l'enfance}

Au fil des années, Jasmine Dubé est devenue une véritable femme-orchestre du thêâtre pour jeunes publics, à la fois auteure, interprète, metteure en scène et directrice de troupe ${ }^{3}$. Loin de se limiter au langage de la scène, elle a également publié des albums ${ }^{4}$ et des romans', participé à l'écriture de manuels scolaires et à la conception de l'émission Passe-Partout ${ }^{6}$. Dans les diverses modalités de son intervention auprès des enfants, on retrouve à chaque fois un même objectif : établir des rapports égalitaires. L'expression est de l'auteure elle-même, qui s'est exprimée sur son travail et sa conception des rapports entre enfants et adultes dans des préfaces et dans des entrevues?

Créée en 1982, Bouches décousues est une commande ${ }^{8}$. Il s'agissait d'écrire une pièce, qui pourrait être jouée dans les écoles, sur les agressions sexuelles subies par les enfants. Comme l'auteure l'indique dans son résumé, la représentation théâtrale a pour but "d'inciter les enfants à parłer". Et elle précise qu'il est "plus que souhaitable. que parents et éducateurs assistent à la pièce qui, "idéalement ", devrait être suivie d'une discussion ( $B D$, p. 7). Cette première pièce a remporté un vif succès. Après 350 représentations au Québec, elle a été jouée en Suisse, en France, en Australie et en Russie ${ }^{9}$. Dans Bouches décousues, les personnages d'adultes parlent d'eux, soit directement au public

3. La troupe Bouches décousues, comme le titre de sa première pièce, fondée en 1986

4. Citons, par exemple, Lourson qui voulait une Juliette (1997). Crattelle au bois mordant (1998) el les albums Elvis (à partir de 2000) en collabotation avec Roger Paré, tous parus à Montréal, La courte échelle, coll. "Il était une fois...".

5. Avant la série des Nazaire, analysée ici, un roman pour pré-adolescents: La tête de Line Hotte, Montréal, Québec/Amérique Jeunesse, 1989.

6. Pour plus d'informations sur la carrière diversifiée de l'auteure, on peut consulter le site Internet: http://www.aqad.qc.ca/pages/auteurs/dube_jasmine. htm.

7. Voir la préface de Petit monstre, Montréal, Leméac, coll. "Théatre Jeunesse", 1993 , p. 7. Voir également Jasmine Dubé, "Faire confiance aux enfants ", Jeu, Montréal, n'76, 1995, p. 92-94.

8. Jasmine Dubé, Bouches décousues, Montréal, Leméac, coll. "Thêâtre pour enfants", 1985. Désormais, les références à cette pièce seront indiquées par le sigle $B D$, suivi de la page, et placées entre parenthèses dans le corps du texte.

9. Voir Diane Pavlovic, "Bouches décousues", Jeu, Montréal, no 76, 1988, p. 116-118. 
par une chanson qui remplit la fonction du monologue classique, soit en s'adressant aux personnages d'enfants. La mère de la fillette révèle son expérience de l'agression sexuelle et les sentiments éprouvés alors. Il est clair que le titre de cette pièce inaugurale, qui deviendra plus tard le nom de la compagnie théâtrale de l'auteure, est à prendre comme une devise, un slogan résumant sa conception des rapports entre les adultes et les enfants.

Des situations et des thèmes de cette pièce seront développés dans la série des Nazaire une dizaine d'années plus tard. Le thème secondaire et le déclencheur de l'action est le sentiment d'envie qu'éprouve l'enfant pour un bien possédé par un autre, sentiment qui évolue rapidement en idée fixe. Sylvie, fille unique, désire avoir un petit frère, alors que Julien, doté quant à lui d'un petit frère, rêve d'avoir une bicyclette comme Sylvie.

Des livres et Zoé, montée en 1987 pour le réseau des Maisons de la culture, a pour objectif, selon le résumé de la pièce, de promouvoir la lecture et l'écriture ${ }^{10}$. Le texte est centré sur l'une des préoccupations majeures de Jasmine Dubé, la solitude de l'enfant, et particulièrement celle de l'enfant unique dont les parents travaillent. La présentation des personnages nous apprend que les parents sont pleins de bonne volonté, mais qu'ils n'arrivent pas à communiquer adéquatement avec leur fille ${ }^{11}$. Lassée des recommandations qu'on lui transmet en laissant de petits billets sur la table de cuisine ou des messages sur le répondeur, celle-ci manifeste son désarroi en faisant une fugue.

Le discours préfaciel des pièces suivantes permet à l'auteure de préciser sa conception du théâtre pour jeunes publics. Dans la préface de Petit monstre (1992), qui met en scène un père et son fils de cinq ans, elle explique qu'elle a voulu écrire une pièce qui s'adresserait aux enfants et à "leurs adultes ${ }^{12}$. L'emploi de ce

10. Jasmine Dubé, Des livres et Zoé: chou bidou woua, Montréal, Leméac, coll. "Thêâtre pour enfants", 1988, p. 9. Désormais, les références à cette pièce seront indiquées par le sigle $L Z$, suivi de la page, et placées entre parenthèses dans le corps du texte.

11. Jasmine Dubé, Des livres et Zoé: cbou bidou woua, ouvr. cité, p. 15. La mère est ainsi décrite: "Elle travaille dans un bureau. Elle tient à avoir une belle relation avec sa fille, mais elle ne sait pas toujours comment s'y prendre". Quant au père, "il est commis comptable. Lui aussi se sent parfois dépassé par l'attitude de Zoé. Pour lui, tout commence par une bonne éducation".

12. Jasmine Dubé, Petit monstre, Montréal, Leméac, coll. "Théâtre Jeunesse", 1993, p. 7. Désormais, les références à cette pièce seront indiquées par le 
possessif est chargé de signification pour Dubé qui, dans Bouches décousues, faisait dire à la petite fille: "On n'en parle pas toujours pour pas faire peur à nos adultes" ( $B D$, p. 43). Les enfants "ont" des adultes, de la même façon que les adultes ont des enfants. Il y a toujours recherche de la réciproque chez la dramaturge, qui écrit ailleurs: "Je veux présenter des situations où l'adulte et l'enfant vivent des rapports égalitaires" $(P M, \mathrm{p} .7)$.

Il s'agissait également pour l'auteure de réagir au courant dominant du père absent et de montrer des rapports de tendresse entre père et fils, de "faire avancer les rapports entre les hommes, les femmes et les enfants. ( $P M$, p. 8). L'action se déroule tôt trop tôt, précise la didascalie ( $P M$, p. 13) - un samedi matin. L'enfant est réveillé avant l'adulte et veut faire jouer ce dernier, qui est partagé entre la tendresse et l'exaspération. L'un des temps forts de la pièce est la scène où père et fils jouent à intervertir leurs rôles; chacun se voit par les yeux de l'autre et trouve que celui-ci exagère. "C'est drôle ça, tu ne trouves pas, dit le père, même si on s'aime, on se fait quand même de la peine" $(P M$, p. 35$)$. La pièce se termine par un renversement de situation: c'est le père, enfin bien réveillé, qui veut jouer avec son fils et lui témoigner son affection, mais celui-ci, après toutes ces émotions, s'est rendormi!

Deux pièces postérieures, Pierrette Pan (1994) et La bonne femme (1996), font ce pari assez insolite dans le théâtre pour la

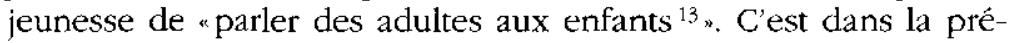
face de Pierrette Pan que l'auteure exprime son désir de "décloisonner", de "raccorder l'adulte et l'enfant" ( $P P$, p. 8). Pierrette Pan aborde l'aspect professionnel de la vie des adultes, le personnage éponyme étant une femme de pouvoir, la ministre de l'enfance et des produits dérivés. Lá ministre préfère malheureusement les produits aux enfants. Affectivement infirme, elle ne peut les supporter, car elle n'a pas su grandir elle-même. À l'opposé, la "bonne femme" est une sans-abri. Cette pièce audacieuse a valu à l'auteure et metteure en scène les prix du Texte original

sigle $P M$, suivi de la page, et placées entre parenthèses dans le corps du texte.

13. Jasmine Dubé, Pierrette Pan, ministre de l'enfance et des produits dérivés, Montréal, Leméac, coll. "Théâtre Jeunesse", 1994, p. 8. Désormais, les références à cette pièce seront indiquées par le sigle $P P$, suivi de la page, et placées entre parenthèses dans le corps du texte. 
et de la Production "Jeunes publics " au Gala des Masques $1996^{14}$.

L'ensemble paratextuel et textuel constitué par les préfaces ou les résumés et le texte même des pièces est très éclairant sur la conception qu'a Jasmine Dubé de ce qu'il est convenu d'appeler le monde de l'enfance. Elle refuse de laisser ce monde clos sur lui-même: puisque les enfants "ont" des adultes, ils ne sauraient être livrés à eux-mêmes ou à la seule société de leurs pairs.

\section{La suite romanesque des Nazaire}

Aussi ne sera-t-on pas surpris que Jasmine Dubé rejette cette convention romanesque qui veut que les parents, exclus du monde de l'enfance, ne soient que des personnages secondaires. La thématique centrale des Nazaire est le fonctionnement de la cellule familiale. Plus qu'une série, les cinq épisodes forment en réalité une suite romanesque qui permet de suivre l'évolution du héros au sein de sa famille durant une période d'environ un an. Fn effet, la série des Nazaire accorde, plus que l'ensemble des romans destinés à cette tranche d'âge, une importance primordiale aux relations entre l'enfant et ses parents ${ }^{15}$. À l'instar des pièces examinées plus haut, ces livres appellent à un dialogue, qui peut être amorcé par une lecture en duo, une mise en voix du texte romanesque.

La collection "Premier roman", où ils sont parus, donne à lire différents types de configuration familiale: un ou deux parents; enfants uniques ou familles de deux ou plusieurs enfants. Dans la majorité des romans, qui se regroupent souvent en séries centrées sur un héros, les parents sont les garants de l'autorité et de la sécurité. Le rôle du confident est généralement joué par un autre adulte (souvent l'un des grands-parents) ou par un ami du même âge. De façon générale, l'adulte intervient auprès de l'enfant sur le mode de l'ordre ou du questionnement, mais ne parle pas de lui-même: l'adulte parle à l'enfant de l'enfant. Certains récits sont

14. Jasmine Dubé, la bonne femme, Montréal, Leméac, coll. "Théâtre Jeunessen, 1997.

15. Ce jugement se fonde sur un dépouillement exhaustif de la collection "Premier roman. (101 titres parus de 1988 à 2000), effectué dans le cadre d'une étude sur les représentations de la masculinité dans le personnage du jeune garçon, à parâitre en 2002 dans Canadian Cbildren's Literature/Littérature canadienne pour la jennesse (CCI/LCJ). Guelph (Ontario). 
axés sur la difficulté pour l'enfant de communiquer avec ses parents, particulièrement avec son père. Quelques exemples permettront de mieux situer les romans de Dubé dans l'ensemble de ceux qui sont offerts à cette classe d'âge.

Dans Adrien n'est pas un chameau de Bertrand Gauthier, à la suite d'une conversation surprise et mal interprétée, le héros éponyme craint de décevoir son père, avec lequel il communique peu. C'est le grand-père qui le rassure et lui redonne l'estime de lui-même. Mais le jeune garçon ne semble pas totalement convaincu et un malaise persiste. Un épilogue en vers reproduit en italique - avatar de la morale des contes et récit didactiques souligne que le problème n'est pas résolu à la fin du récit: "Il serait donc bon pour Adrien/De parler avec son père Urbain " "." Dans Les mots font la grève de Jean-Marie Poupart, l'enfant se dit en panne de mots parce qu'il est fâché contre son père. Ce dernier, peu préoccupé de rétablir la communication, décrète: "Il y a des choses qu'un enfant ne peut pas comprendre ${ }^{17}$ " $(M G$, p. 50); à l'opposé, la mère multiplie les efforts, jugeant la situation angoissante ( $M G, \mathrm{p}, 22)$. Victime d'une réaction allergique, l'enfant finit par comprendre qu'il faut exprimer son désaccord, car les mots retenus l'ont empoisonné: "Si on ne s'en sert pas, les mots pourrissent. (MG, p. 60). Pas de prison pour Chausson de Gilles Gauthier constitue un autre exemple pertinent du traitement de cette problématique dans le roman pour jeunes lecteurs. Le père d'un ami y confie au héros les difficultés qu'il éprouve à communiquer avec son fils. Il est frappant de remarquer que, dans ces trois romans parus en 1999, c'est le fils qui doit faire le chemin vers le père et non l'inverse.

Le contexte précisé, nous voici plus à même de définir la teneur particulière des Nazaire. Les cinq romans, parus de 1994 à 1999, constituent un ensemble de quelque trois cents petites pages, imprimées en gros caractères. La date de publication du premier roman est postérieure de deux ans à la première représentation de Petit monstre. Ce n'est sans doute pas un hasard si le

16. Bertrand Gauthier, Adrien n'est pas un chameau, Montréal, La courte échelle, coll. "Premier roman", n' 85, 1999, p. 62.

17. Jean-Marie Poupart, Les mots font la grève, Montréal, La courte échelle, coll. "Premier roman ", n" 84, 1999, p. 50 . Désormais, les références à ce roman seront indiquées par le sigle $M G$, suivi de la page, et placées entre parenthèses dans le corps du texte. 
discours préfaciel de cette pièce, dont nous avons cité des extraits plus haut - décrire une relation de tendresse entre père et fils, faire avancer les rapports entre enfants et adultes, montrer des rapports égalitaires - semble tout à fait illustré par le projet romanesque des Nazaire. L'histoire est celle d'un manque que ressent le héros - un petit frère ou une petite soeur - manque qui est comblé, mais dont la satisfaction entraîne chez l'enfant des sentiments inattendus et exige une renégociation de sa place dans la famille. On retrouve ici le thème de la solitude de l'enfant unique face au bloc que forment les adultes, et qui était abordé dans les premières pièces de Dubé. Nazaire exprime explicitement ce sentiment: "Toi et Maman, vous êtes des adultes. Moi, je suis un enfant tout seul comme un pauvre petit microbe ${ }^{18}$." Ce manque est ressenti par l'enfant comme une injustice.

La situation de départ est similaire à celle de la première pièce de l'auteure, Bouches décousues. Le personnage enfant est aux prises avec un sentiment dévastateur pour la sérénité et l'estime de soi. De même que Sylvie et Julien s'enviaient mutuellement, Nazaire envie un enfant de sa classe, Boris, qui a tout. La première phrase de Fais un voeu, Nazaire!, qui émane de la voix narratoriale, donne le ton: "Il y en a qui sont chanceux!" (FV, p. 7). Pour empêcher l'enfant de se laisser empoisonner par ce sentiment délétère, l'aide de l'adulte est essentielle. Dès la première page, intervient la parole maternelle rapportée: "- Pense à quelque chose que tu as et que Boris n'a pas, dit ma mère " $(F V$, p. 7). En adoptant cette stratégie, Nazaire en vient à découvrir qu'il possède une chienne, Caramel. Mais il se souvient alors que cette chienne n'est qu'un pis-aller, un succédané. Il a reçu de ses parents un bébé chien alors qu'il souhaitait un bébé tout court.

Une fois le problème de Nazaire identifié, le premier épisode (Fais un vou, Nazaire!) propose une rétrospective destinée à rappeler un échec de la famille - une fausse-couche de la mère - et se termine par l'annonce d'une nouvelle grossesse. Tout en suivant la progression de la grossesse et en décrivant les changements qu'elle provoque dans la chimie des relations entre les membres de la famille, le deuxième épisode (Nazaire et les mous-

18. Jasmine Dubé, Fais un vceu, Nazaire!, Montréal, La courte échelle, coll. "Premier roman ", $\mathrm{n}^{\circ} 35,1994$, p. 29. Désormais, les références à ce roman seront indiquées par le sigle $F V$, suivi de la page, et placées entre parenthèses dans le corps du texte. 
quetaires) explore l'univers extrafamilial, l'école et les amis de la famille. Le troisième épisode (Les deux Nazaire) est axé sur la relation de l'enfant et du grand-père, qui portent le même prénom. Quant au quatrième (Tu n'es plus seul, Nazaire), il met l'accent sur la transformation de la cellule familiale, symbolisée par l'emménagement dans une nouvelle maison. Enfin, dans le dernier épisode (Le grand Nazaire), l'enfant exprime et apprend à dépasser la jalousie suscitée par l'attention accordée au nouveau venu. Bien qu'on voie l'enfant de sept ans évoluer à l'école et avec ses amis, l'intrigue est nettement familiocentrique. Outre le narrateur, les trois personnages importants de la suite romanesque sont le père, la mère et le grand-père maternel.

\section{Les dialogues enfant-adultes}

L'analyse se centre sur les dialogues entre l'enfant et l'un des parents, parfois les deux, qui occupent une place significative dans cette suite romanesque. Dans chacun des épisodes, qui comportent six ou sept chapitres et parfois un épilogue, il y a au moins une conversation importante entre Nazaire et l'un des parents ou le grand-père. À cela, il faut ajouter des échanges souvent plus brefs entre Nazaire et le clan des adultes, c'est-à-dire une voix parentale qui n'est pas toujours identifiée.

Examinons la structure de Fais un vou, Nazaire!, dans lequel est posé le problème du jeune garçon : la solitude face au bloc des parents. Lá visée est rétrospective et, en introduction, le narrateur raconte les problèmes posés par l'arrivée dans la famille de la chienne Caramel, succédané du bébé désiré. Les fragments de dialogue insérés dans la narration rapportent des échanges assez vifs entre les deux voix parentales et Nazaire:

[...] C'était fatigant. Mes parents me chicanaient sans arrêt.

- Si tu rangeais tes jouets, Caramel ne les grugerait pas.

- Ferme la porte de ta chambre, elle ne pourra pas entrer.

- Si vous m'aviez donné un vrai bébé, aussi! ( $F V$, p. 14)

On notera que, dans ce chassé-croisé de reproches et de conseils, aucune des parties ne prend la responsabilité de la situation.

Dans le chapitre 2 qui raconte la fausse-couche de la mère survenue quelques années auparavant, le point culminant consiste en un échange entre les trois membres de la famille réunis dans une chambre d'hôpital. Devant la question de l'enfant: 
"Pourquoi?", la mère avoue son ignorance et ne décourage pas les hypothèses de son fils. Quand il suggère que le bébé s'est envolé vers les étoiles, elle répond: "Peut-être bien" (FV 22). Le milieu du livre - les chapitres 3 et 4 - est consacré à deux têteà-tête, d'abord avec le père, ensuite avec la mère. Le chapitre 5 présente le troisième interlocuteur important de la suite romanesque: le grand-père. La voix narratoriale observe qu'à la différence des parents, le grand-père ne pose pas de questions, mais que l'enfant lui raconte toujours tout. Le dernier chapitre fait intervenir le milieu de vie extrafamilial, l'école. L'enfant annonce à la maîtresse que sa mère attend un nouveau bébé. Enfin, un épilogue élucide le titre: Nazaire fait le voeu que le petit pois (c'est le nom qu'il donne à l'embryon) s'accroche bien cette fois ( $F V$, p. 61).

L'arrivée de la chienne Caramel, dans le premier épisode, préfigure les difficultés que causera, dans le dernier épisode, l'arrivée du petit frère. C'est dans ce dernier épisode, où il s'agit de surmonter la crise causée par cette arrivée, que les échanges verbaux sont les plus nombreux.

L'attaque des dialogues présente une caractéristique digne de mention. En effet, un examen systématique révèle que, dans la grande majorité des cas, la parole de l'adulte est la première, qu'il s'agisse d'une voix parentale non identifiée, du père ou, surtout, de la mère. Il ne faudrait pas pour autant conclure à une dominance de l'adulte sur l'enfant. À la différence de la parole théâtrale, la parole romanesque est une parole rapportée, filtrée par la voix narratoriale. Dans le discours des parents, Nazaire sélectionne ce qui répond bien, mal ou médiocrement à ses attentes. Cette parole elliptique, qui parvient au lecteur par la médiation du narrateur enfant, ne peut être reçue qu'avec le support de la narration. Dans le cas de Dubé, tout se passe comme si le roman permettait à lauteure dramatique non seulement de commenter et d'interpréter les dialogues, mais aussi de développer les propositions contenues dans les préfaces et dans les nombreuses didascalies de ses pièces.

Dans beaucoup de romans jeunesse contemporains, les dialogues ont pour fonction principale de faire avancer l'action ${ }^{19}$. Ils

19. Voir Ganna Ottevaere-van Praag, Le roman pour la jeunesse. Approcbes definitions - techniques narratives, Berne, Peter Lang, 1996, p. $71-72$. 
sont plus informatifs que didactiques, moraux ou introspectifs. Dans les Nazaire, bien au contraire, les dialogues ont pour objet la confrontation des points de vue des principaux protagonistes.

\section{Fonctions des dialogues}

Dans le cadre restreint de cet article, les principales fonctions des dialogues sont présentées de façon schématique et illustrées par un ou quelques exemples.

\section{L'enfant communique le problème}

Nazaire exprime son sentiment de solitude et d'injustice. Après un gros cauchemar doublé d'un pipi au lit, il se plaint à son père:

- Ce n'est pas juste. Toi et maman, vous êtes deux adultes. Moi, je suis un enfant tout seul comme un pauvre petit microbe. J'ai seulement Caramel.

- C'est déjà ça de pris. Il y a tout plein d'enfants qui n'ont pas de chien tu sais.

- Toi, quand tu étais petit, tu en avais, un chien. Et tu avais quand même des frères et sœeurs. Mäis pas moi. Quand je vais être un père, moi, je vais leur en faire, des frères et des sceurs à mes enfants, tu vas voir ${ }^{20}(F V$, p. 29-30).

Le père emploie sans succès la stratégie du "pense à ce que tu as", utilisée par la mère dans les premières pages. Ici, le dernier mot est laissé à l'enfant. La réponse paternelle se manifeste par la gestuelle: "Mon père me serre fort. Ça me fait tout doux en dedans" (FV, p. 30).

\section{L'enfant définit le type de communication convenable}

Dans Fais un vou, Nazaire!, la mère vient chercher l'enfant à l'école pour aller manger au restaurant. Elle a pris l'initiative de ce moment d'intimité, mais elle accepte les règles du jeu que propose Nazaire :

20. Le personnage de fille de Bouches décousues disait à peu près la même chose à sa mère: "Moi plus tard, quand j'vas être grande, $j$ 'vas leur en faire des bébés à mes enfants. ( $B D, \mathrm{p} .33)$. 
- D'accord.

Je l'aime, ma mère, quand elle est comme ça. Quand elle ne dit pas: "Nazaire, ne fais pas ci. Nazaire, ne fais pas ça." Non, aujourd'hui, elle dit :

- Nazaire, j'aime ça être avec toi.

Je lui souris.

- Je taime, maman, même si des fois, je te trouve tannante.

- Je t'aime, Nazaire, même si des fois je te trouve tannant ( $F V$. p. 36-37).

Un échange ludique suit cet échange verbal. Le parallélisme des constructions, indiquant la réciprocité, est un procédé caractéristique de l'écriture de Dubé qui veut donner à lire la confrontation des points de vue, des "rapports égalitaires". C'est aussi une variante sur le "ce n'est pas parce qu'on s'aime qu'on ne se fait pas mal", thème de Petit monstre. La symétrie des répliques satisfait le désir de justice que le narrateur exprime de façon récurrente.

Cette symétrie sert aussi à montrer qu'enfants et adultes sont sur la même longueur d'onde. L'adulte, en indiquant qu'il vit une expérience similaire à celle de l'enfant, prouve qu'il le comprend. Ainsi, quand Nazaire dit à sa mère qu'il est "tanné. de se faire demander toutes les deux minutes s'il est content d'avoir un petit frère ou une petite sceur, celle-ci lui répond:

- Moi aussi, les gens me font des commentaires: "Tu es grosse. Tu vas avoir des jumeaux. Fais attention à ceci ou cela." Ouf, j'ai l'impression d'avoir une bedaine publique ${ }^{21}$.

Et quand le père et le fils se lèvent en retard un matin, Nazaire juge normal d'écrire un billet d'excuse pour son père :

- [...] Puis je vais te faire un billet de retard. Zut! Moi aussi, je suis en retard.

- Ne t'en fais pas, papa, je vais te faire un billet de retard, moi aussi (FV, p. 33-34).

Beaucoup de dialogues ludiques exercent une fonction analogue, renforçant la complicité entre les adultes et les enfants. Les

21. Jasmine Dubé, Tu n'es plus seul, Nazaire!, Montréal, La courte échelle, coll. "Premier roman", n" 57, 1997, p. 32-33. Désormais, les références à ce roman seront indiquées par le sigle $P S$, suivi de la page, et placées entre parenthèses dans le corps du texte. 
envies singulières que suscite la grossesse chez la mère constituent un sujet de plaisanterie qui court tout le long des épisodes; celle-ci accepte les plaisanteries et participe même aux échanges, renchérissant sur l'extravagance de ses goûts alimentaires: "Confiture de poulet avec des cerises, du vinaigre et de la ratatouille ${ }^{22}{ }^{\text {. }}$

\section{L'enfant et l'adulte accordent leur langage}

Les quiproquos que provoque une utilisation différente du langage constituent un thème important du thêâtre et du roman de l'auteure. Dans Des livres et Zoé, elle expliquait ce qu'était une figure de style ( $L Z$, p. 47-48). L'enfant prend ces figures au sens premier. Des expressions usées pour l'adulte suscitent chez lui des images vives et parfois intolérables. Ainsi quand la mère dit que le bébé est gros comme une tête d'épingle, la comparaison inquiète Nazaire car les épingles piquent ( $F V$, p. 19-20). Quand plus tard elle parle de la taille d'un petit pois, cette image déclenche une fantasmagorie chez l'enfant, qui imagine des nuées de petits pois voyageant dans les étoiles. Quand le père dit que Nazaire était à croquer quand il était petit, la voix narratoriale commente: "Hin, hin, très drôle. On ne croque pas les bébés, quand même" (PS, p. 15-16).

Souvent, la création verbale est un moyen pour l'enfant de s'approprier le langage. Nazaire dit à sa mère: "tu m'as mis un pou dans l'oreille , et celle-ci corrige: "la puce à l'oreille, Nazaire. Pas un pou dans l'oreille ${ }^{23}$,. Enfin, il arrive qu'il corrige les mots des adultes à cause de ce qu'ils révèlent sur les attitudes. À plusieurs reprises, dans les différents épisodes, il reprend sa mère qui utilise le possessif mon en parlant du bébé.

- C'est mon bébé à moi.

— NOTRE bébé! Notre bébé à NOUS!

- Bien sûr, Nazaire!

22. Jasmine Dubé, Nazaire et les mousquetaires, Montréal, La courte échelle, coll. "Premier roman", n" 39, 1995, p. 63. Désormais, les références à ce roman seront indiquées par le sigle $N M$, suivi de la page, et placées entre parenthèses dans le corps du texte.

23. Jasmine Dubé, Les deux Nazaire, Montréal, La courte échelle, coll. "Premier roman ", n 50,1996, p. 21-22. Désormais, les références à ce roman seront indiquées par le sigle $D N$, suivi de la page, et placées entre parenthèses dans le corps du texte. 
Et plus loin:

- Il a faim, mon petit amour...

- NOTRE petit amour, Maman ${ }^{24}$ !

\section{L'enfant apprend à négocier}

Il est remarquable que les parents disent plusieurs fois dans le récit: "D'accord Nazaire". Les dialogues rapportent les négociations pour partager la même chambre que le petit frère, puis pour la décoration de celle-ci. La voix du narrateur signale que Nazaire, par son plaidoyer, a remporté une grande victoire.

Mes parents sont désemparés.

Ils se regardent.

- Je n'avais jamais vu ça de cette façon, mais...

- Oui... c'est un point de vue... ajoute mon père.

- Évidemment, si tu penses que...

- D'accord.

Youpi! Il y a des jours où ça vaut la joie d'argumenter avec les adultes (PS, p. 16-17).

Plus loin, à l'issue d'une autre discussion, le grand-père complimente Nazaire: "Tu te débrouilles plutôt bien dans les négociations, mon écureux" (PS, p. 20-21).

\section{L'adulte dévoile ses problèmes et ses faiblesses à l'enfant}

La mère n'hésite pas à expliquer à l'enfant les changements émotionnels qu'occasionne la grossesse. Elle laisse Nazaire proposer ses explications:

- Peut-être que tu es contente pour deux, ou triste pour deux.

- Peut-être ( $D N$, p. 35).

24. Jasmine Dubé, Le grand Nazaire, Montrêal, La courte échelle, coll. "Premier roman ", $\mathbf{n}^{\circ} 87,1999$, p. 12-13. Désormais, les références à ce roman seront indiquées par le sigle $G N$, suivi de la page, et placées entre parenthèses dans le corps du texte. 
Nazaire ayant le trac avant un exposé, son père lui confie, au grand étonnement de l'enfant, que lui non plus n'aime pas parler en public ( $P S$, p. 40 ). L'effet de cet échange est lisible quelques pages plus tard, quand le narrateur se dit réconforté en sachant que son père pense à lui pendant l'exposé ( $P S, \mathrm{p}$. 44). Dans le dernier épisode, le père révèle à Nazaire les sentiments de jalousie qu'il a éprouvés, enfant, à la naissance de sa sœur ( $G N$, p. 52-53).

\section{L'adulte dit à l'enfant ce qu'il pense de lui.}

Cette dernière caractéristique me paraît être l'une des grandes réussites des Nazaire. L'adulte livre son appréciation soit à l'intéressé, soit à une tierce personne en présence de l'enfant. Par exemple, dans le dernier chapitre de la série, intitulé "Journée de mamours avec maman", les sentiments maternels sont alternativement exprimés par le discours indirect et indirect libre rapporté par la voix narratoriale et par le discours direct. Puis la voix narratoriale commente ces paroles.

Elle dit que, depuis quelque temps, elle ne me voit plus. Et elle est jalouse parce que mon père et moi passons beaucoup de temps ensemble.

- Moi aussi, j’ai envie d'être seule avec mon grand garçon d'amour.

Son "grand garçon d'amour"! C'est moi, ça! (GN, p. 55).

Dans le troisième épisode, la mère lit à Nazaire son journal de grossesse où elle s'adresse au bébé: "Tu sais, bébé, ton grand frère Nazaire, c'est vraiment quelqu'un de bien" ( $D N$, p. 13). Après ces interventions parentales, le narrateur enfant voit son estime de soi raffermie, tout comme son importance aux yeux de ses parents.

\section{Un monde partagé}

Le théâtre comme le roman de Jasmine Dubé optent pour un monde partagé par les enfants et les adultes, plutôt que pour un monde de l'enfance dont les parents se tiendraient à distance. Son théâtre laissait entrevoir une tentation romanesque dans la précision que l'auteure apportait aux didascalies et dans les titres qu'elle donnait aux scènes ${ }^{25}$. À l'inverse, ses romans destinés à la

25. Petit monstre constitue un excellent exemple à cet égard. 
même tranche d'âge me semblent convier leur lectorat à un théâtre intimiste. Les conditions de réception optimales des Nazaire ne seraient pas fournies par la lecture solitaire et silencieuse, mais par une lecture conjointe parent-enfant. Les dialogues, dont je viens de citer quelques exemples, offrent à l'enfant et à l'adulte une mise en paroles de situations familiales délicates. Dans le dernier épisode, l'auteure indique d'ailleurs subrepticement le mode d'emploi des textes quand son narrateur mentionne, à l'heure du coucher: "Puis je lis un chapitre de mon livre avec mon père" ( $G N$, p. 45). Quant au signal sonore qui ponctue chaque fin de chapitre - Abracadabreu, Abracadabrouche, Abracadabrune, etc., selon la dernière syllabe du texte - n'indique-t-il pas, très adéquatement, la fin de la séance de lecture à deux? 